\title{
Mapping the neural circuitry of predator fear in the nonhuman primate
}

In rodents, innate and learned fear of predators depends on the medial hypothalamic defensive system, a conserved brain network that lies downstream of the amygdala. However, it remains unknown whether this network is involved in primate fear. Here we demonstrate that visually evoked predator fear recruits a homologous medial hypothalamic defense system in the nonhuman primate

\section{Affiliations}

${ }^{1}$ Shenzhen Key Lab of Neuropsychiatric Modulation and Collaborative Innovation Center for Brain Science, Guangdong Provincial Key Laboratory of Brain Connectome and Behavior, CAS Center for Excellence in Brain Science and Intelligence Technology, Brain Cognition and Brain Disease Institute (BCBDI), Shenzhen Institutes of Advanced Technology, Chinese Academy of Sciences, Shenzhen-Hong Kong Institute of Brain Science-Shenzhen Fundamental Research Institutions, Shenzhen 518055, China

${ }^{2}$ Australian Regenerative Medicine Institute, Monash University, Clayton, VIC 3800, Australia

${ }^{3}$ Epigenetics \& Neurobiology Unit, EMBL Rome, European Molecular Biology Laboratory, Via Ramarini 32, 00015 Monterotondo (RM), Italy

* To whom correspondence should be addressed: gross@embl.it, 


\section{MAIN TEXT}

\section{Introduction}

Lesions of the amygdala block the processing of learned and innate fear stimuli in multiple species, including humans (1-4). However, fear induced by internally generated stimuli, such as the inhalation of carbon dioxide, do not require the amygdala (5). This observation suggests that circuits downstream of the amygdala are sufficient to sustain the behavioral and emotional correlates of fear. This view is supported by extensive work in rodents demonstrating that a circuit from the amygdala to the medial hypothalamus and brainstem, called the medial hypothalamic defensive system, is both necessary and sufficient for innate and learned defensive responses to predators (610). Furthermore, in humans, direct electrical stimulation of the ventromedial hypothalamus $(\mathrm{VMH})$ is sufficient to elicit feelings of intense fear and trigger panic attacks, suggesting that the medial hypothalamus may also participate in human fear (11). Unlike rodents, however, primates typically depend exclusively on visual cues to detect and respond to innate threat cues, yet it remains unknown whether such stimuli are sufficient to recruit the medial hypothalamic defensive system and by what pathway this might occur.

Here we developed a method to elicit robust predator-evoked escape responses in a nonhuman primate (marmoset monkey) under controlled laboratory conditions, and used c-Fos mapping combined with anatomical tract tracing to determine the neural circuits involved. The common marmoset, Callithrix jacchus, is an appealing primate species to study the link between neural circuits and behavior because of its small size and its rich repertoire of affiliative and agonistic social behaviors (12). The defensive responses of marmosets to predators in the wild have been described and include visual scanning, alarm calling, mobbing, avoidance, freezing, and flight $(13,14)$. Exposure to toy snakes, cats or raptors have been used in the laboratory setting to induce visual scanning, alarm calling, freezing and threat displays (15-17). However, to the best of our knowledge, the full repertoire of active defensive responses, including flight and post-flight vigilance observed in response to predators in the wild, has not been reported under laboratory conditions. 
Results

\section{Snake presentation evokes flight, sustained avoidance, and vigilance in the primate}

Animals were pre-trained over a two-month period to enter a transparent plexiglass transport box juxtaposing the home cage. This was followed by 2-4 weeks of habituation to a testing room, during which time the animal was given access via a small door in the transport box to an opaque nest box (Fig. 1AB). Following habituation, the animals underwent surgery for injection of fluorescent anterograde (dextran amine) and retrograde (cholera toxin B) tracers into the VMH (Fig. 2B). Recent advances in MRI-guided stereotaxic surgery in the marmoset(18) have enabled the precise delivery of reagents to discrete brain regions. Following recovery, the animals were trained for an additional five days, during which time their baseline behaviors in the isolated testing room were recorded. On the following day, a remotely activated canopy positioned in front of the transport box was raised to reveal the threat stimulus - a moving rubber snake, while control animals were exposed to a neutral stimulus - a small black box (Fig. 1B), for a continuous duration of 45 minutes (Fig S1A). Exposure to the threat stimulus elicited robust defensive responses in all animals, consisting of flight to the rear of the cage and hypervigilance, and culminating in escape to the adjacent nest box (Fig. 1BC, Video S1). The behavioral state of the animal was scored using a Defensive Behavior Index, consisting of a weighted sum of distance from the stimulus (from -0.8 when near the stimulus to +1 when in the retreat box) and defensive behavior (from -1 when grooming to +2 during flight; Fig. 1C). As long as the threat stimulus was visible, animals remained principally in the nest box (Fig. 1D, Pre $=4.3 \%$ vs Threat $=93 \%, \mathrm{P}<0.0001, \mathrm{t}=14.69$, $\mathrm{df}=8$ ), showing a significant reduction in exploration $($ Fig. 1E, Pre $=6.0$ vs Threat $=1.2, \mathrm{P}<0.05, \mathrm{t}=$ 2.75, $\mathrm{df}=8)$ and increase in lurking (Fig. 1F, Pre $=0.0$ vs Threat $=2.1, \mathrm{P}<0.01, \mathrm{t}=4.75, \mathrm{df}=8$ ) and staring (Fig. 1G, Pre $=0.04$ vs Threat $=1.2, \mathrm{P}<0.05, \mathrm{t}=2.78, \mathrm{df}=8$ ). Scanning, on the other hand, was not significantly affected by threat presentation $($ Fig. $\mathbf{1 H}$, Pre $=0.48$ vs Threat $=0.39$ ). In comparison, animals exposed to the control stimulus demonstrated no signs of startle or flight (Fig. S1B, Video S2), and mainly remained outside of the retreat box with no change in exploration (Fig. S1B-E). On the contrary, control animals stood closer to the front of the transport box to observe the stimulus (Fig. S1F, Pre $=0.1$ vs $\mathrm{Stim}=3.0, \mathrm{P}<0.01, \mathrm{t}=29$, $\mathrm{df}=2$ ), while scanning behaviors remained unchanged (Fig. S1G). In a subset of animals $(\mathrm{N}=3)$ the threat stimulus was presented for five minutes and then hidden again under the canopy to assess recovery (Fig. S2A). Following removal of the threat stimulus, animals exited the nest box and their Defensive Behavior Index returned to baseline (latency $=43-120 \mathrm{sec}$, Fig. S2B,C). During this recovery period, animals 
91 spent significantly less time in the retreat box (Fig. S2D, Threat $=90 \%$ vs Recovery $=38 \%, P<$ $920.05, \mathrm{t}=3.67, \mathrm{df}=4)$, but other behaviors such as lurking, staring and scanning did not return to 93 baseline levels (Fig. S2E-H). A subset of animals was exposed for 45 minutes to either the threat $94(\mathrm{~N}=2)$ or neutral stimulus $(\mathrm{N}=2)$ and processed for histological analysis and cFos expression 95 (Fig. 1A, Fig. S1A).

96 Threat exposure recruits the primate medial hypothalamic defensive system

To determine whether the medial hypothalamus and its efferent and afferent targets are recruited in primates following predator exposure, we performed immunostaining for cFos - an immediateearly gene, in brain sections from experimental and control animals that had previously undergone injection of anterograde and retrograde tracers unilaterally into VMH (Fig. 2B, right). Within $\mathrm{VMH}, \mathrm{cFos}+$ cells were detected in the dorsomedial division (VMHdm), the subnucleus known to be essential for predator defense in rodents and whose activation is sufficient for the induction of fear and panic in humans (11) (Fig. 2A left, Fig. S3A,C). Importantly, no cFos + cells were detected in VMH of control animals (Fig. S3B) or in the ventrolateral division of VMH (VMHvl) known to mediate defensive responses to social threat $(8,19)$._Consistent with similar studies in rodents $(20)$ cFos + cells were also detected prominently in experimental, but not control animals in the dorsal periaqueductal grey (dPAG; Fig. 2A right, Fig. S4), a region known to be required for expression of predator defense in rodents $(21,22)$. Finally, we identified cFos + cells in the arcuate and paraventricular hypothalamic nuclei (Fig. S3AB), although similar numbers of immunopositive cells were seen in experimental and control animals, suggesting generalized recruitment of these structures during behavioral testing. Due to the high immunoreactive background, we were unable to determine the extent of cFos labeling in forebrain areas such as amygdala or medial prefrontal cortex (mPFC).

Anterograde and retrograde tracer delivery were restricted to $\mathrm{VMH}$ in three of four animals, allowing us to identify afferent and efferent regions of this structure in the primate brain and compare them with similar studies in the rodent. Sparse retrograde tracer-labeled cell bodies were found in the ventral mPFC (Fig 2B left, Fig. S5AB), medial division of the bed nucleus of the stria terminalis (BNST; Fig 2B middle, Fig. S5C), ventral division of the medial and lateral septum (respectively MS and LS; Fig 2B middle, Fig. S5C), posterior basomedial amygdala (BMA; Fig. 2B right, Fig. S6A,CD), and basolateral amygdala (BLA; Fig. S6A,B,D). Dense retrograde tracerlabeled cell bodies were found in the ventral division of the medial amygdala (MeA; Fig. 2B right; Fig. S6A,D,E), consistent with this structure providing significant inputs to VMH in rodents (23). 
Major anterograde tracer-labeled axonal varicosities, on the other hand, were found in the periaqueductal grey (PAG; Fig. 2C, Fig. S7AB,F) and sparse anterograde label was found in the medial pulvinar (PM; Fig. 2C, Fig. S7A-C), intermediate layer of the superior colliculus (SGI; Fig. 2C, Fig. S7A-B,D) and medial pretectal nucleus (MPN; Fig. 2C, Fig. S7_AB,E). A few anterograde varicosities were also visible in MeA, BNST, and LS, but these could not be reliably confirmed.

\section{Discussion}

The mechanism by which the amygdala is recruited and how it might influence defensive behavior remains contested $(1,24)$. It is proposed that visually-evoked defensive responses to predators depends on fast, brainstem information processing via retino-collicular projections $(24,25)$. From there, threat information passes to mesencephalic motor initiation centers to drive fixed medullary motor programs $(26,27)$. The importance of this pathway is confirmed by the observation that SC lesions in primates abrogate both orienting and anxiety responses to a predator $(28,29)$. At the same time, amygdala lesions also block fear and anxiety responses to predators in primates and humans $(3,4)$. Here we show that the medial hypothalamic defensive system, known to be essential for defensive responses to predator in rodents (6-8), is engaged during predator defense in the marmoset.

This finding has two important implications for our understanding of fear in humans. First, our discovery makes it likely that the medial hypothalamus plays a similar role in encoding an internal state of threat in primates as it does in rodents (10,21,30-32). Notably, VMHdm in rodents is required for the induction and expression of both innate and conditioned predator fear $(8,10,21)$ and stimulation of VMHdm in monkeys and humans is sufficient to elicit an intense defensive emotional state $(11,33)$. The conserved recruitment of medial hypothalamus across species means that these structures must be considered in the search for the etiology and therapeutic treatment of anxietyrelated disorders in humans.

Second, our identification of conserved connectivity of the medial hypothalamic defensive system across rodents and primates supports a common function for this system in defensive behaviors. In particular, the discovery that the primate $\mathrm{VMH}$ also receives major inputs from MeA, a nucleus known to convey information from the accessory olfactory system in rodents $(34,35)$, was unexpected as this sensory system is vestigial in primates (36). These results suggest that MeA may have elaborated its non-olfactory inputs as vision evolved to become the dominant sense in primates. Finally, the existence of inputs to VMH from SC and MPN offers a direct pathway for 
156 visual information to rapidly enter the medial hypothalamic defensive system (Fig. 2D) $(34,37)$

157 Nevertheless, given that amygdala lesions block fear responses to predators in humans ${ }^{3}$, a parallel, 158 indirect route that brings visual information to forebrain structures and from there to the medial 159 hypothalamus is likely to also be important for both the emotional and behavioral responses to 160 visual threat. In summary, our data argue for a conserved role of medial hypothalamic instinctive 161 behavior networks across mammals, including humans. Further work is required to assess precisely 162 which aspects of defensive behavior they control and whether they are necessary for generating the conscious emotional states that accompany threat in humans.

Materials \& Methods

Animals

Six Common Marmosets (Callithrix jacchus) aged 18-24 months were sourced from the Australian National Nonhuman Primate Breeding and Research Facility. All experiments were conducted in accordance with the Australian Code of Practice for the Care and Use of Animals for Scientific Purposes and were approved by the Monash University Animal Ethics Committee, which also monitored the welfare of these animals.

\section{Behavioral assay}

Testing was conducted in a transparent plexiglass transport box (305 x 295 x $205 \mathrm{~mm})$ connected by a circular opening to a detachable black plexiglass nest box $(600 \times 140 \times 130 \mathrm{~mm})$ and placed on a table in the testing room in front of a removable black cloth canopy that covered the threat and control stimuli, respectively, a black striped rubber toy snake and a square black cardboard box. The snake could be animated by manipulating a pulley system from outside the isolated experimental room. On the day of the stimulation, a separate pulley system was used to lift the canopy, rapidly revealing the stimulus without the need for the experimenter to enter the room. A camera (GoPro Hero4) was positioned in front of the transport box to record the animal's behavior. Manipulation of the stimulus and monitoring of the animal were all conducted in a room separate from the animal's experimental room. The experimenter remained out of sight of the animals in a different room during testing. All animals underwent initial training for habituation to the experimental room. During this period, the animals were trained to enter the transport box that had been mounted to their home cage. Once the animals were habituated to the transport box, they were transported daily to the experimental room for habituation. There, the transport box was connected 
to the nest box, and the animals were allowed to explore the apparatus freely. The duration of habituation sessions was gradually increased from a few minutes to 20 minutes until the animal remained calm and relaxed during the entire session. At the end of each training session, a reward was given according to the animal's preference before being brought back to their home cage. All training sessions commenced at 15:00 hours. Total time of transport box training and experimental room habituation was approximately two months. Once all animals were similarly habituated, four animals underwent tracer injection surgery and, following seven days of recovery, an additional 5 days of experimental room habituation to reinforce the training (20 minutes per session). Following retraining of the animals were exposed to either the control $(\mathrm{N}=2)$ or threat stimulus $(\mathrm{N}=2)$. Optimization of the control and threat stimulus presentation was conducted with the animal that did not undergo tracer surgery. Experimental validation involved testing behavioral responses to the threat and control stimuli and assessing recovery $(\mathrm{N}=3,5$ minutes each: free apparatus exploration, stimulus exposure, stimulus occlusion, free apparatus exploration). The animals were randomly assigned to threat $(\mathrm{N}=2$, one male, one female) or control $(\mathrm{N}=2$, one male, one female) stimulus conditions (5 minutes free exploration, 45 minutes stimulus exposure). Following testing, the animal was rapidly processed for histological analysis.

\section{Behavior assessment}

Videos were scored offline using BORIS software (38). The experimental space was divided into 6 zones: close vs far from the stimulus, standing vs close to the ground, and head out vs head in while in the retreat box. A weighted Defensive Behavior Index was calculated from these scores and represented as a continuously varying measure or heatmap. Exploration was estimated by counting the passage of the animal between zones. For several measures, data from both groups of animals were included as the protocols were indistinguishable over the first ten minutes of testing. Lurking was considered as both peeking (body in the retreat box, head out) and body and head in the retreat box while keeping the threat in sight. Staring was scored by counting each time the animal looked at the stimulation area. Scanning was scored by counting each time the animal looked upwards and around. All behaviors were analyzed by unpaired t-test.

\section{Surgery}

Preparation of the animals for MRI-guided microinjection of the VMH was performed as previously described (18). In brief, animals were anaesthetized and scanned in a $9.4 \mathrm{~T}$ small-bore animal scanner. To facilitate reconstruction of the marmoset brain and visualization of the VMH structural 
T2 images were acquired with parameters included the following - repetition time/echo time: 6,000/40 ms, echo train length: 4, field of view: $38.4 \times 38.4 \mathrm{~mm}^{2}$, acquisition matrix: $192 \times 192$, 100 coronal slices adjusted according to the size of the brain, slice thickness: $0.4 \mathrm{~mm}$, signal averages: 4, scan time: 19 min, 42 s. Subsequently, the left hemisphere VMH was pressure injected with $180 \mathrm{nl}$ of a bi-directional neural tracer cocktail consisting of $5 \mu \mathrm{g} / \mu \mathrm{l}$ retrograde Cholera toxin subunit B conjugated with Alexa Fluor 488 (Life Technologies, cat\# C22841) and $50 \mu \mathrm{g} / \mu \mathrm{l}$ anterograde dextran amine 10,000 MW conjugated with Alexa Fluor 488 (Life Technologies, cat\# D22910). Animals were allowed one week to recover to facilitate transport of neural tracers. Following seven days of recovery, animals underwent behavioral testing.

\section{Tissue processing}

232 At the conclusion of behavioral testing, animals were deeply anaesthetized with $100 \mathrm{mg} / \mathrm{kg}$ sodium 4\% paraformaldehyde in 0.01M PBS. Brains were post-fixed for $24 \mathrm{hrs}$ in 4\% PFA before being serially dehydrated in sucrose $(10 \%, 20 \%$, and $30 \%)$ solutions before being snap-frozen in $-50 \mathrm{C}$ isopentane and cryosectioned in the coronal plane at $50 \mu \mathrm{m}$. Sections were divided into four series and stored free-floating in a cryoprotective solution consisting of 50\% phosphate buffered saline (PBS), 30\% ethylene glycol and 20\% glycerol at -20 C. For each subject, a full series was mounted onto glass slides (Superfrost plus), dehydrated in serial alcohols and cleared in xylene before being mounted in DPX for analysis of tracer label.

\section{Histology and immunohistochemistry}

Tissue sections were rinsed in PBS before undergoing pre-treatment in a blocking solution consisting of PBS with 10\% normal donkey serum and 2\% Triton-X for $1 \mathrm{~h}$ at room temperature. Following blocking sections were incubated with primary antibody in pre-treatment solution for 16-18 hrs at 4 C. Sections were then washed in PBS before incubation in donkey anti-rabbit Alexa Fluor 594 secondary antibody (1:1,000, Life Technologies, cat\# A11058) for $1 \mathrm{~h}$ at room temperature and washed and counterstained with Hoechst (Life Technologies, cat\# H3569). Primary antibodies used in this study were rabbit anti-cFos (1:1,000, Sigma Aldrich, cat\# F7799) to assess neural activity and rabbit anti-parvalbumin (1:2,000, Swant cat\# PV27) to delineate boundaries of the superior colliculus. Acetylcholinesterase staining was performed to allow for demarcation of amygdala subnuclei and layers of the superior colliculus. The staining protocol was adapted from previous studies(39). 


\section{Microscopy and image processing}

254 Imaging was performed on an Axio Imager Z1 microscope (Zeiss). Images were acquired with a 255 Zeiss Axiocam HRm digital camera using Axiovision software (v. 4.8.1.0). The objectives used 256 were Zeiss EC-Plan Neofluar 5x0.16, \#420330-9901, EC-Plan Neofluar 10x0.3, \#420340-9901; 257 Plan Apochromat 20x0.8 \#420650-9901; EC Plan Neofluar 40x1.3 oil \#420462-9900. Filter sets 258 used were Zeiss DAPI \#488049-9901-000, Zeiss HE eGFP \#489038-9901-000 and Zeiss HQ TR $259 \quad \# 000000-1114-462$. Stitching of images and adjustments to contrast and brightness were 260 performed using Adobe Photoshop CC2015. The line art, boundaries and contours for all figures 261 were executed using Adobe Illustrator CC2015. Demarcation of areas was achieved with AChE 262 and parvalbumin labeling and compared with the Marmoset Brain in Stereotaxic Coordinates(40). 


\section{References}

1. LeDoux JE. Coming to terms with fear. Proceedings of the National Academy of Sciences. $2014 \mathrm{Feb}$ 25;111(8):2871-8.

2. Anderson DJ, Adolphs R. A Framework for Studying Emotions across Species. Cell. 2014 Mar;157(1):187200.

3. Feinstein JS, Buzza C, Hurlemann R, Follmer RL, Dahdaleh NS, Coryell WH, et al. Fear and panic in humans with bilateral amygdala damage. Nat Neurosci. 2013 Mar;16(3):270-2.

4. Martinez RC, Carvalho-Netto EF, Ribeiro-Barbosa ÉR, Baldo MVC, Canteras NS. Amygdalar roles during exposure to a live predator and to a predator-associated context. Neuroscience. 2011 Jan;172:314-28.

5. Feinstein JS, Adolphs R, Damasio A, Tranel D. The Human Amygdala and the Induction and Experience of Fear. Current Biology. 2011 Jan;21(1):34-8.

6. Canteras NS. The medial hypothalamic defensive system: hodological organization and functional implications. Pharmacol Biochem Behav. 2002 Mar;71(3):481-91.

7. Gross CT, Canteras NS. The many paths to fear. Nature Reviews Neuroscience. 2012;13(9):651-658.

8. Silva B a, Mattucci C, Krzywkowski P, Murana E, Illarionova A, Grinevich V, et al. Independent hypothalamic circuits for social and predator fear. Nature neuroscience. 2013;16(November):1731-3.

9. Wang L, Chen IZ, Lin D. Collateral Pathways from the Ventromedial Hypothalamus Mediate Defensive Behaviors. Neuron. 2015 Mar;85(6):1344-58.

10. Kunwar PS, Zelikowsky M, Remedios R, Cai H, Yilmaz M, Meister M, et al. Ventromedial hypothalamic neurons control a defensive emotion state. eLife. 2015 Mar 6;4:e6633.

11. Wilent WB, Oh MY, Buetefisch CM, Bailes JE, Cantella D, Angle C, et al. Induction of panic attack by stimulation of the ventromedial hypothalamus. JNS. 2010 Jun;112(6):1295-8.

12. Miller CT, Freiwald WA, Leopold DA, Mitchell JF, Silva AC, Wang X. Marmosets: A Neuroscientific Model of Human Social Behavior. Neuron. 2016 Apr;90(2):219-33.

13. Ferrari SF, Ferrari MAL. Predator avoidance behaviour in the buffy-headed marmoset,Callithrix flaviceps. Primates. 1990 Jul;31(3):323-38.

14. Corrêa HKM, Coutinho PEG. Fatal attack of a pit viper,Bothrops jararaca, on an infant buffy-tufted ear marmoset (Callithrix aurita). Primates. 1997 Apr;38(2):215-7.

15. Vitale AF, Visalberghi E, De Lillo C. Responses to a snake model in captive crab-eating macaques (Macaca fascicularis) and captive tufted capuchins (Cebus apella). International Journal of Primatology. 1991 Jun;12(3):27786.

16. Barros M, Boere V, Huston JP, Tomaz C. Measuring fear and anxiety in the marmoset (Callithrix penicillata) with a novel predator confrontation model: effects of diazepam. Behav Brain Res. 2000 Mar;108(2):20511.

17. Shiba Y, Kim C, Santangelo AM, Roberts AC. Lesions of either anterior orbitofrontal cortex or ventrolateral prefrontal cortex in marmoset monkeys heighten innate fear and attenuate active coping behaviors to predator threat. Front Syst Neurosci [Internet]. 2015 Jan 21 [cited 2019 May 28];8. Available from: http://journal.frontiersin.org/article/10.3389/fnsys.2014.00250/abstract

18. Mundinano I-C, Flecknell PA, Bourne JA. MRI-guided stereotaxic brain surgery in the infant and adult common marmoset. Nat Protoc. 2016 Jul;11(7):1299-308.

19. Wang L, Talwar V, Osakada T, Kuang A, Guo Z, Yamaguchi T, et al. Hypothalamic Control of Conspecific Self-Defense. Cell Reports. 2019 Feb;26(7):1747-1758.e5.

20. Baisley SK, Cloninger CL, Bakshi VP. Fos expression following regimens of predator stress versus footshock that differentially affect prepulse inhibition in rats. Physiology \& Behavior. 2011 Oct;104(5):796-803.

21. Silva BA, Mattucci C, Krzywkowski P, Cuozzo R, Carbonari L, Gross CT. The ventromedial hypothalamus mediates predator fear memory. European Journal of Neuroscience. 2016;43(11):1431-1439.

22. de Andrade Rufino R, Mota-Ortiz SR, De Lima MAX, Baldo MVC, Canteras NS. The rostrodorsal periaqueductal gray influences both innate fear responses and acquisition of fear memory in animals exposed to a live predator. Brain Struct Funct. 2019 May;224(4):1537-51.

23. Canteras NS, Simerly RB, Swanson LW. Organization of projections from the medial nucleus of the amygdala: A PHAL study in the rat. J Comp Neurol. 1995 Sep 18;360(2):213-45.

24. Pessoa L, Adolphs R. Emotion processing and the amygdala: from a "low road" to "many roads" of evaluating biological significance. Nat Rev Neurosci. 2010 Nov;11(11):773-82.

25. Carr JA. I'll take the low road: the evolutionary underpinnings of visually triggered fear. Front Neurosci [Internet]. 2015 Oct 29 [cited 2019 May 28];9. Available from: http://journal.frontiersin.org/Article/10.3389/fnins.2015.00414/abstract

26. Apps R, Hawkes R, Aoki S, Bengtsson F, Brown AM, Chen G, et al. Cerebellar Modules and Their Role as Operational Cerebellar Processing Units. Cerebellum. 2018 Oct;17(5):654-82.

27. Koutsikou S, Apps R, Lumb BM. Top down control of spinal sensorimotor circuits essential for survival: Midbrain control of sensory and motor systems. J Physiol. 2017 Jul 1;595(13):4151-8. 
28. Maior RS, Hori E, Barros M, Teixeira DS, Tavares MCH, Ono T, et al. Superior colliculus lesions impair threat responsiveness in infant capuchin monkeys. Neuroscience Letters. 2011 Oct;504(3):257-60.

29. DesJardin JT, Holmes AL, Forcelli PA, Cole CE, Gale JT, Wellman LL, et al. Defense-Like Behaviors Evoked by Pharmacological Disinhibition of the Superior Colliculus in the Primate. Journal of Neuroscience. 2013 Jan 2;33(1):150-5.

30. Krzywkowski P, Penna B, Gross CT. Dynamic encoding of social threat and spatial context in the hypothalamus [Internet]. Neuroscience; 2019 Oct [cited 2019 Nov 29]. Available from: http://biorxiv.org/lookup/doi/10.1101/811380

31. Masferrer ME, Silva BA, Nomoto K, Lima SQ, Gross CT. Differential encoding of predator fear in the ventromedial hypothalamus and periaqueductal grey [Internet]. Neuroscience; 2018 Mar [cited 2019 Nov 29]. Available from: http://biorxiv.org/lookup/doi/10.1101/283820

32. Kennedy A, Kunwar PS, Li L, Wagenaar D, Anderson DJ. Stimulus-specific neural encoding of a persistent, internal defensive state in the hypothalamus [Internet]. Neuroscience; 2019 Oct [cited 2019 Nov 29]. Available from: http://biorxiv.org/lookup/doi/10.1101/805317

33. Lipp HP, Hunsperger RW. Threat, Attack and Flight Elicited by Electrical Stimulation of the Ventromedial Hypothalamus of the Marmoset Monkey Callithrix jacchus; pp. 260-275. Brain Behav Evol. 1978;15(4):260-75. 34. Swanson LW, Petrovich GD. What is the amygdala? Trends in Neurosciences. 1998 Aug;21(8):323-31. 35. Yao S, Bergan J, Lanjuin A, Dulac C. Oxytocin signaling in the medial amygdala is required for sex discrimination of social cues. eLife. 2017 Dec 12;6:e31373.

36. Trotier D. Vomeronasal organ and human pheromones. European Annals of Otorhinolaryngology, Head and Neck Diseases. 2011 Sep;128(4):184-90.

37. Wei P, Liu N, Zhang Z, Liu X, Tang Y, He X, et al. Processing of visually evoked innate fear by a noncanonical thalamic pathway. Nat Commun. 2015 Nov;6(1):6756.

38. Friard O, Gamba M. BORIS: a free, versatile open-source event-logging software for video/audio coding and live observations. Fitzjohn R, editor. Methods Ecol Evol. 2016 Nov;7(11):1325-30.

39. Paul CA, Beltz B, Berger-Sweeney J. Staining for Acetylcholinesterase in Brain Sections. Cold Spring Harb Protoc. 2010 Aug;2010(8):pdb.prot4806.

40. Paxinos G, Watson C, Petrides M, Rosa M, Tokuno H. The marmoset brain in stereotaxic coordinates. 2012. 


\section{Acknowledgements}

General: We thank Claire Warner for launching the project and helping to delineate regions of the marmoset $\mathrm{VMH}$ and MeA.

Funding: The work was supported by EMBL and Monash University, the European Research Council (ERC) Advanced Grant COREFEAR to C.T.G. and J.A.B. is supported by a Senior Research Fellowship support (APP1077677) from the National Health and Medical Research Council (NHMRC), and L.W. is supported by the International Partnership Program of Chinese Academy of Sciences (172644KYS820170004) and National Science Foundation of China (91732304).

Author contributions: All the behavioral experiments and their analysis were carried out by Q.M. Surgical experiments were performed by W.K. and J.A.B. Histological experiments and analysis were carried out by W.K and Q.M. C.T.G. and J.A.B. conceived the project and with Q.M. and W.K. designed the experiments. C.T.G., J.A.B., Q.M and W.K. wrote the manuscript.

Competing interests: There are no competing interests.

\section{Figure Legends}

Figure 1. Snake presentation evokes flight, sustained avoidance, and vigilance in the primate. (A) Following an extensive period of habituation training animals underwent surgery for the local delivery of anterograde and retrograde tracers in $\mathrm{VMH}$. After recovery training was continued for 5 days before exposing the animal to the experimental stimulus. Forty-five minutes after stimulus exposure the animal was anesthetized, perfused, and its brain prepared for cFos immunostaining. (B) Animals were randomly assigned to groups exposed to either an animated rubber snake (threat) or a black cardboard box (control). Images indicate representative behaviors evoked by the stimuli during the initial and persistent response phases of the test. (C) Defensive Behavior Index (light color, individual traces; dark color, mean; $60 \mathrm{sec}$ bins) for animals in the threat (red) and control (blue) groups during the final training (pre-test), baseline (pre-stimulus) and stimulus exposure (stimulus) sessions. Threat exposure induced a significant (D) increase in time spent in the retreat box, (E) decrease in exploration, $(\mathbf{F})$ increase in lurking, and $(\mathbf{G})$ increase in staring, but no significant $(\mathbf{H})$ change in scanning (mean of first 5 minutes; $\mathrm{N}=5 ; * * * * \mathrm{P}<0.0001, * * \mathrm{P}<0.01$, $* \mathrm{P}<0.05)$.

Figure 2. Threat exposure recruits the primate medial hypothalamic defensive system. (A) Robust cFos immunostained cells were found in the dorsomedial VMH and dorsalmedial PAG in animals exposed to snake threat, but not those exposed to the control stimulus (red dots indicate cFos + cells in representative animal; $\mathrm{N}=2$ ). (B) Retrograde and anterograde tracing revealed sparse VMH afferents from mPFC, BNST, LS, MS, BMA and BLA and dense inputs from MeA. (C) Dense VMH efferents were found in PAG and sparse outputs in PM. (D) Summary of the VMH afferents and efferents in the primate. Dark grey arrows denote pathways that were identified in this study.

\section{Supplementary Figure Legends}


Figure S1. Behavior of animals in the cFos experiment. (A) Experimental phases: 5 min prestimulus during which the animal was freely exploring the apparatus, 45 min stimulus when the animal was exposed to the threat (toy animated snake) or control (black box) stimulus by raising a black cloth canopy, and post-experiment processing where the animal was anesthetized and perfused for histology and cFos immunistaining. (B) Defensive behavior for each animal in the threat (top) and control (bottom) groups. Color code indicates the animal is hiding in the retreat box (cold) or exploring (warm). (C-G) Quantification of behaviors of threat (red) and control (blue) groups during the pre-stimulus vs stimulus periods $(5 \min$ bins, $\mathrm{N}=2-5$; $* * * * \mathrm{P}<0.0001$, $* * \mathrm{P}<$ $0.01, * \mathrm{P}<0.05)$.

Figure S2. Behavior of animals in the recovery experiment. (A) Experimental phases: $5 \mathrm{~min}$ pre-stimulus during which the animal was freely exploring the apparatus, 5 min stimulus when the animal was exposed to the threat (toy animated snake) stimulus by raising a black cloth canopy, and recovery period when the stimulus was covered again by the cloth canopy. (B) Defensive behavior for each animal. Color code indicates the animal is hiding in the retreat box (cold) or exploring (warm). (C) Defensive Behavior Index (light color, individual traces; dark color, mean; $60 \mathrm{sec}$ bins) for animal during the pre-test, pre-stimulus, threat, and recovery phases. (D-H) Quantification of behaviors during the pre-threat, threat, and recovery phases $(\mathrm{N}=3 ; * * \mathrm{P}<0.01$, $* \mathrm{P}<0.05)$.

Figure S3. Threat-evoked cFos immunolabel in VMH. Representative coronal sections of the marmoset brain showing cFos immunolabeling in VMH of animals exposed to the (A) threat or (B) control stimulus. Green asterisk denotes needle tract from tracer administration. (C-E) High powered images from insets showing cFos + cells identified within the $\mathrm{VMH}$. No cFos + cells were identified in animals exposed to the control stimulus. The arcuate (Arc) and paraventricular nucleus of the hypothalamus (PVH) contained cFos + cells in both threat and control animals (numbers indicate animal ID; A, B: scale $=200 \mu \mathrm{m}$; C-E: scale $=20 \mu \mathrm{m})$.

Figure S4. Threat-evoked cFos immunolabel in PAG. Representative coronal sections of the marmoset brain showing cFos immunolabeling in dPAG of animals exposed to the (A) threat or (B) control stimulus. (C-E) High powered images from insets showing cFos + cells identified within the dPAG. No cFos + cells were identified in animals exposed to the control stimulus (numbers indicate animal ID; A, B: scale $=200 \mu \mathrm{m}$; C-E: scale $=20 \mu \mathrm{m})$.

Figure S5. VMH connectivity in the forebrain. Representative coronal sections of the marmoset brain into which retrograde and anterograde tracers were delivered into VMH showing retrograde labeled cell bodies in (A, B high resolution inset) medial prefrontal cortex (mPFC) and (C) bed nucleus of the stria terminalis (BNST) and lateral and medial septum (LS and MS, respectively; scale: A $500 \mu \mathrm{m}, \mathrm{B} 100 \mu \mathrm{m}, \mathrm{C} 200 \mu \mathrm{m})$.

Figure S6. VMH connectivity in amygdala. Representative coronal sections of the marmoset brain into which retrograde and anterograde tracers were delivered into VMH showing retrograde labeled cell bodies in (A, high resolution insets B-C) BLA and BMA and (D, high resolution inset E) MeA (grayscale inset shows acetylcholinesterase stained adjacent section that was used to delineate brain regions; scale: A $1000 \mu \mathrm{m}, \mathrm{B}-\mathrm{C}$ and E $50 \mu \mathrm{m}, \mathrm{D} 100 \mu \mathrm{m})$.

Figure S7. VMH connectivity in midbrain and thalamus. Representative coronal sections of the marmoset brain into which retrograde and anterograde tracers were delivered into VMH showing anterograde labeled processes in (A-B low resolution images with insets $\mathbf{C}-\mathbf{F}$; AChE and Parvalbumin to delineate anatomical boundaries) PAG and PM, and retrograde labeled cell bodies in the SGI and MPN (scale: C $50 \mu \mathrm{m}, \mathrm{D}-\mathrm{E} 20 \mu \mathrm{m}$ and F $100 \mu \mathrm{m}$ ).

\section{Supplementary Materials}


Fig. S1. Behavior of animals in the cFos experiment.

Fig. S2. Behavior of animals in the recovery experiment.

Fig. S3. Threat-evoked cFos immunolabel in VMH.

Fig. S4. Threat-evoked cFos immunolabel in PAG.

Fig. S5. VMH connectivity in the forebrain.

Fig. S6. VMH connectivity in the amygdala.

Fig. S7. VMH connectivity in the midbrain and thalamus.

Movie S1. Animal response to threat stimulation.

Movie S2. Animal response to neutral stimulation. 
A

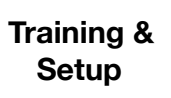

1.5 months

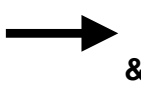

\&
Surgery

$$
3 \text { days }
$$

Habituation

5 days
Stimulus presentation \& Perfusion

$45 \mathrm{~min}$

B

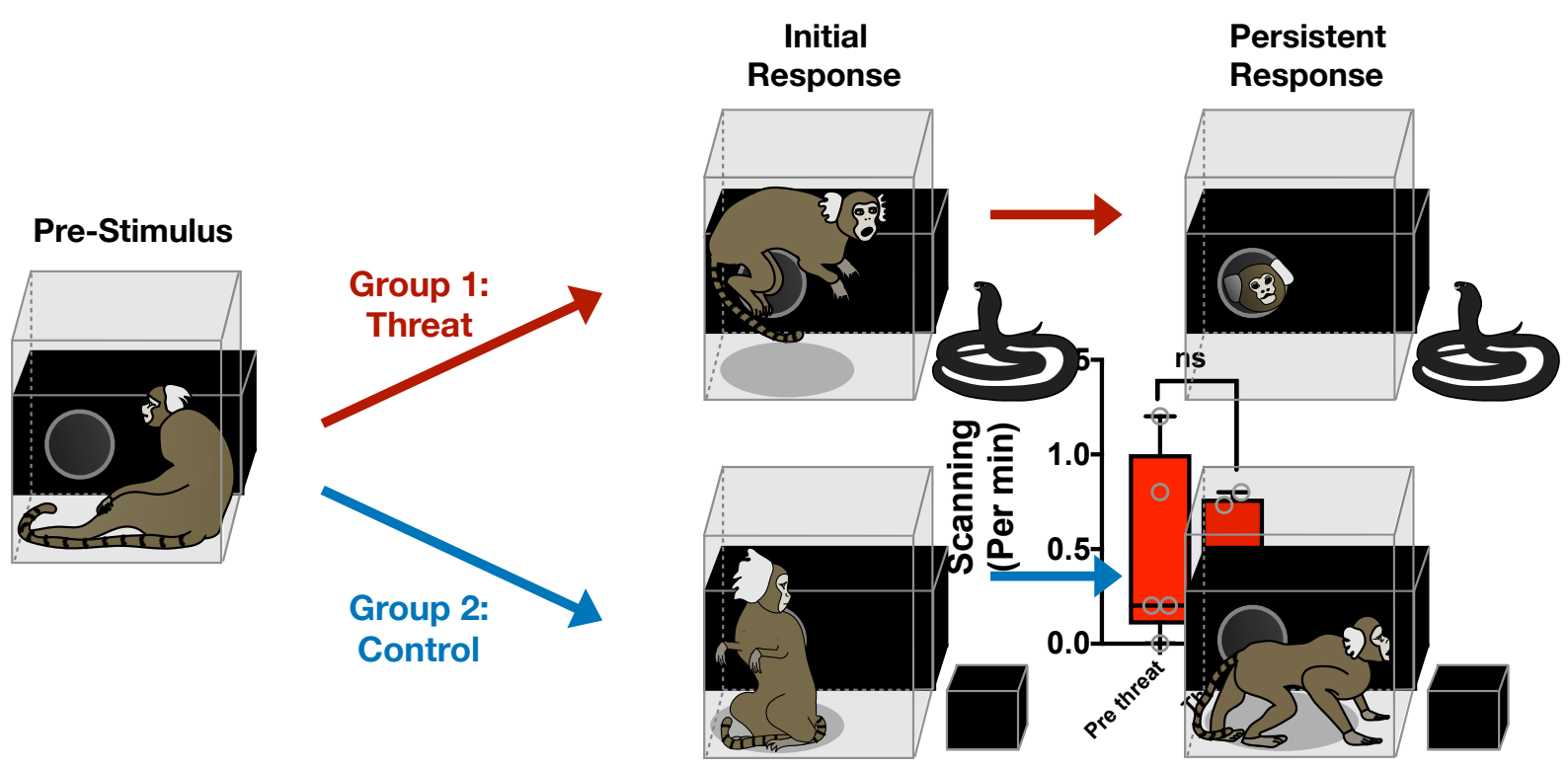

C

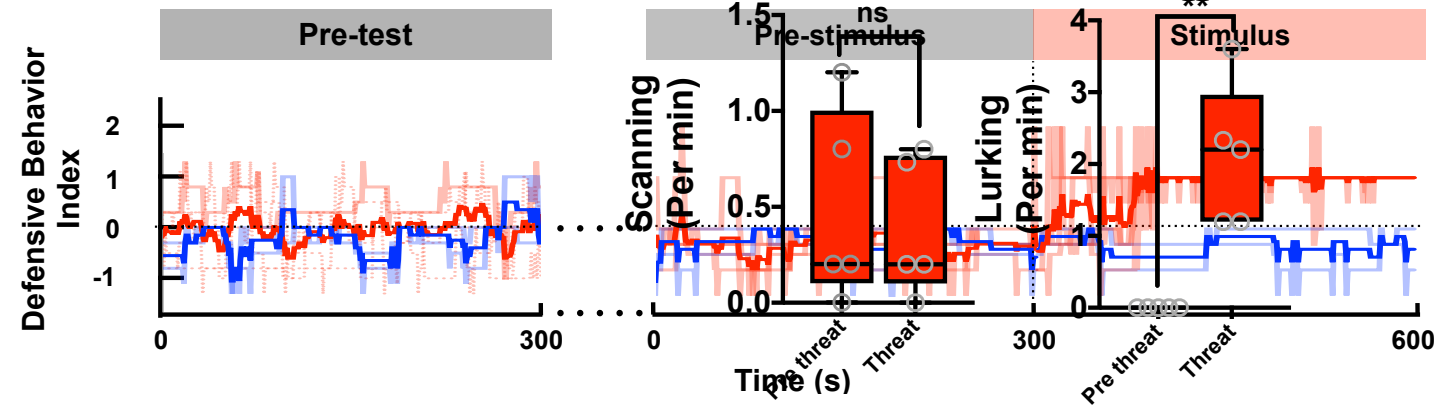

D

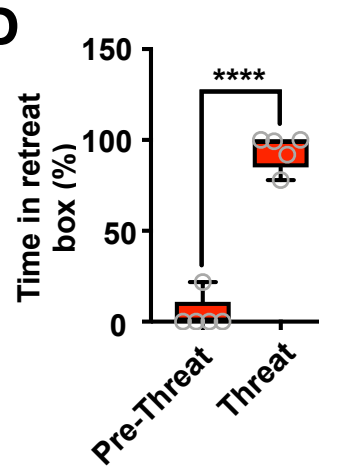

E

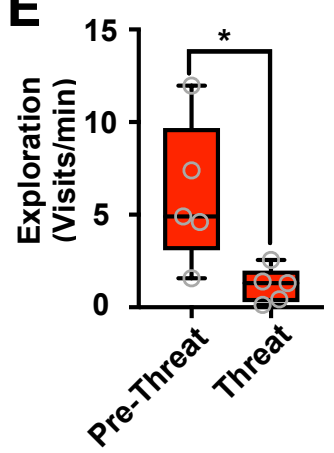

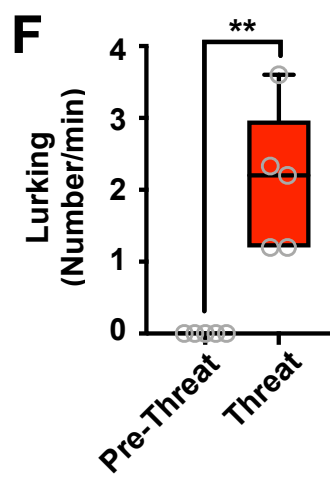

G

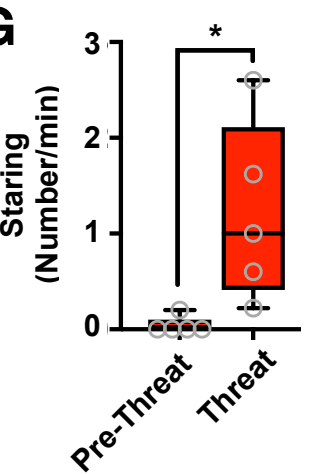

H

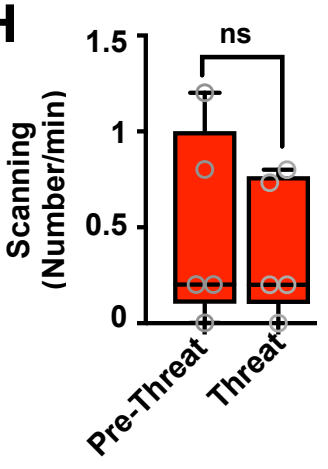


Fig. 2
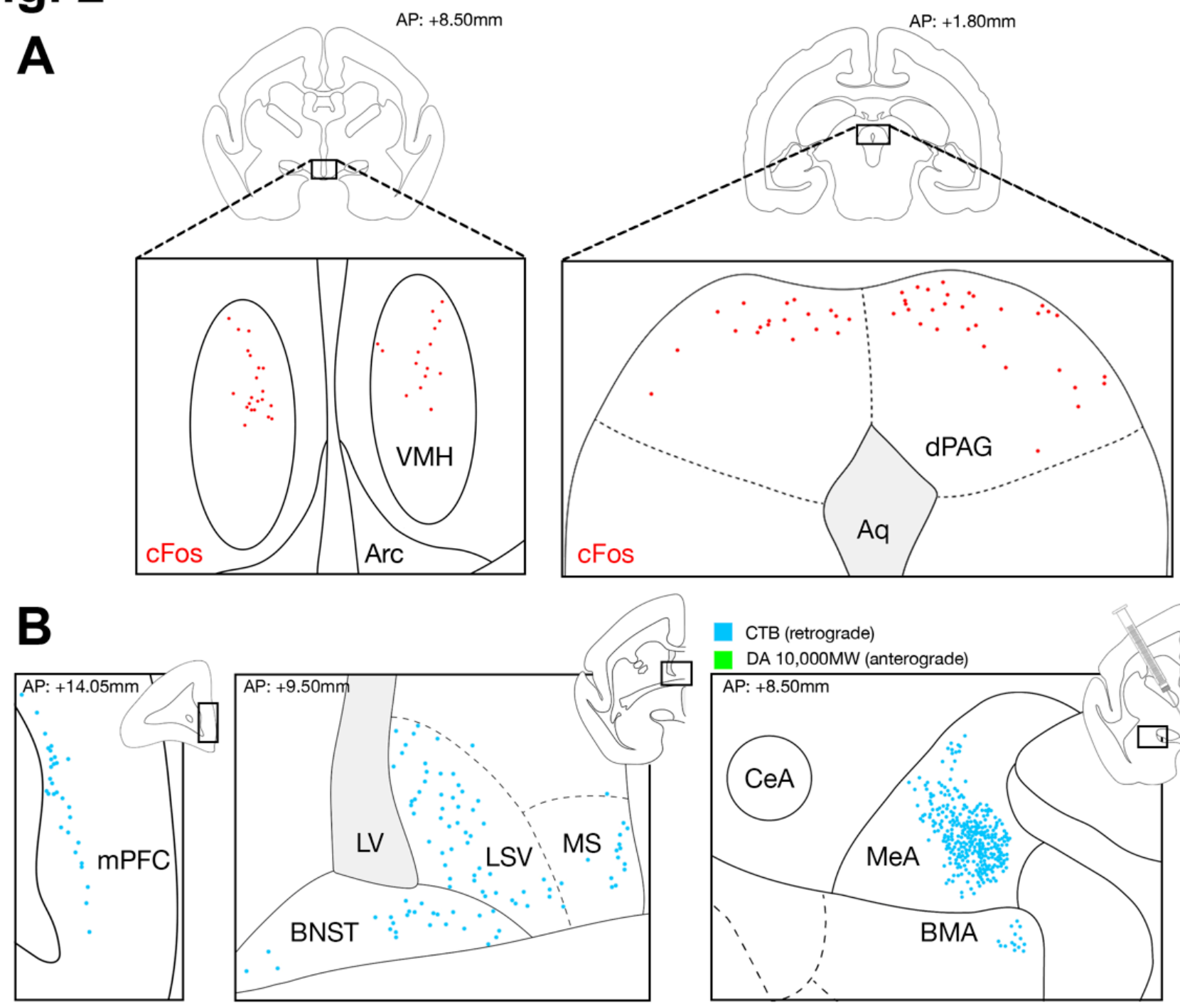

\section{C}

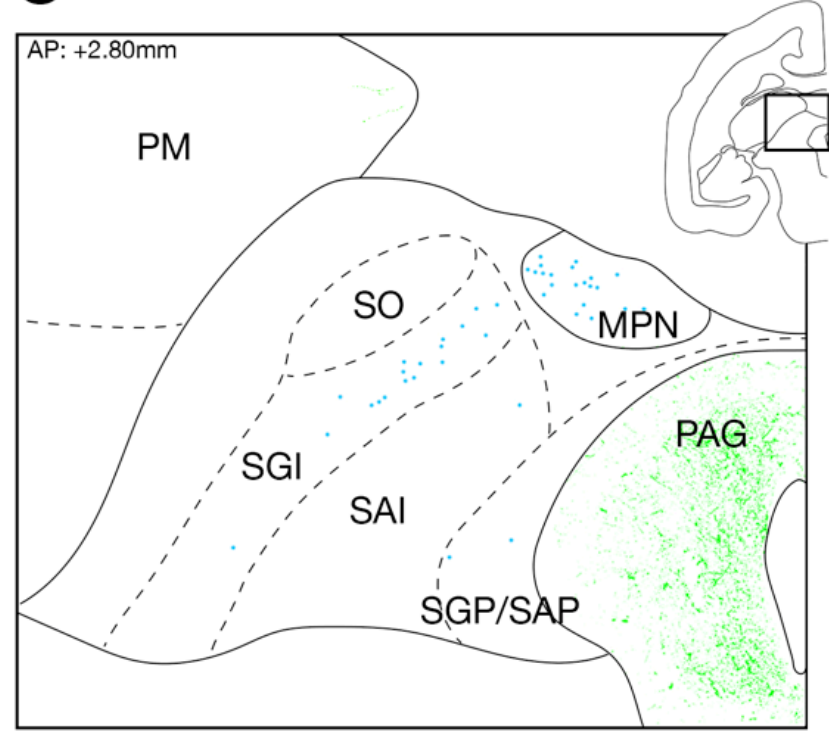

CTB (retrograde)

DA 10,000MW (anterograde)

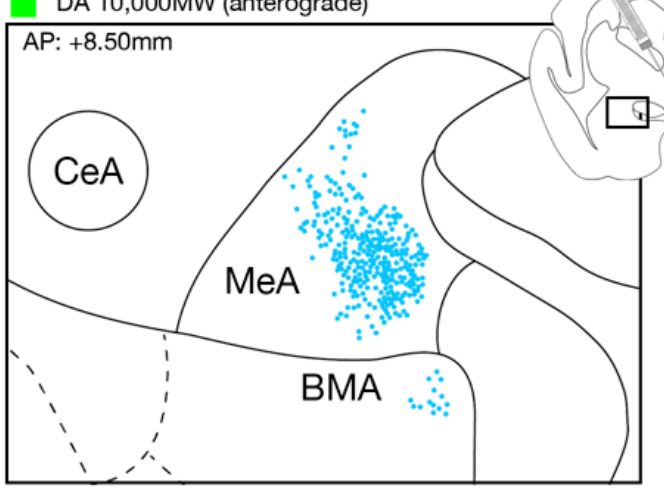

D

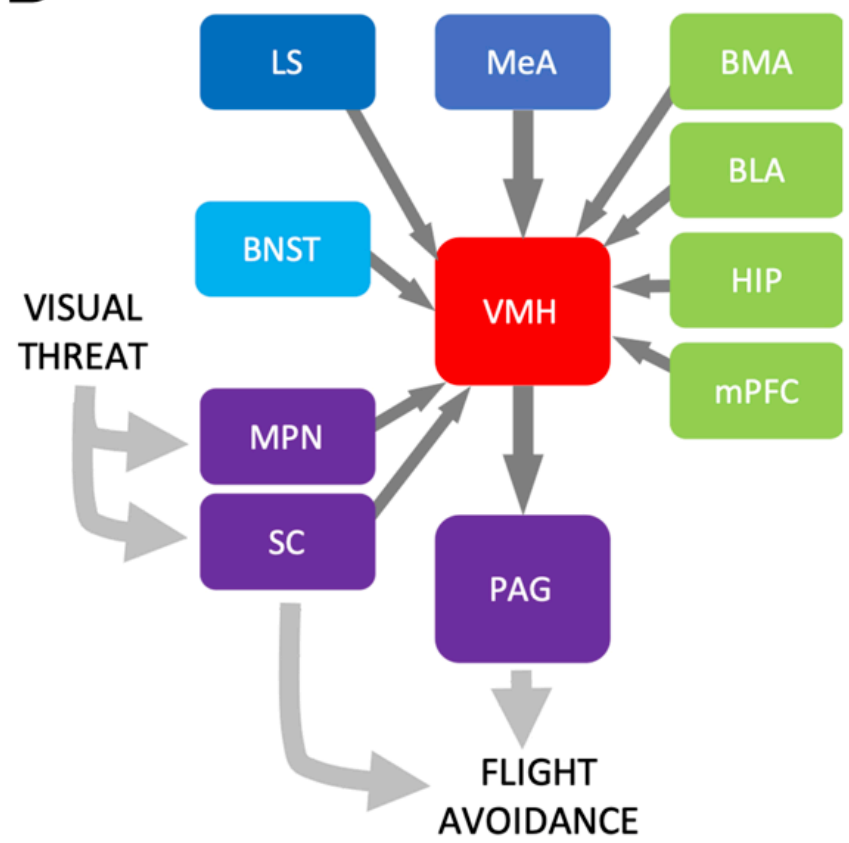




\section{Fig. S1}

A

Stimulus

45 min

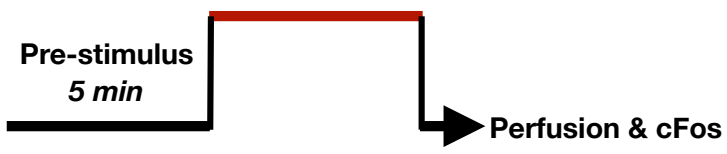

B
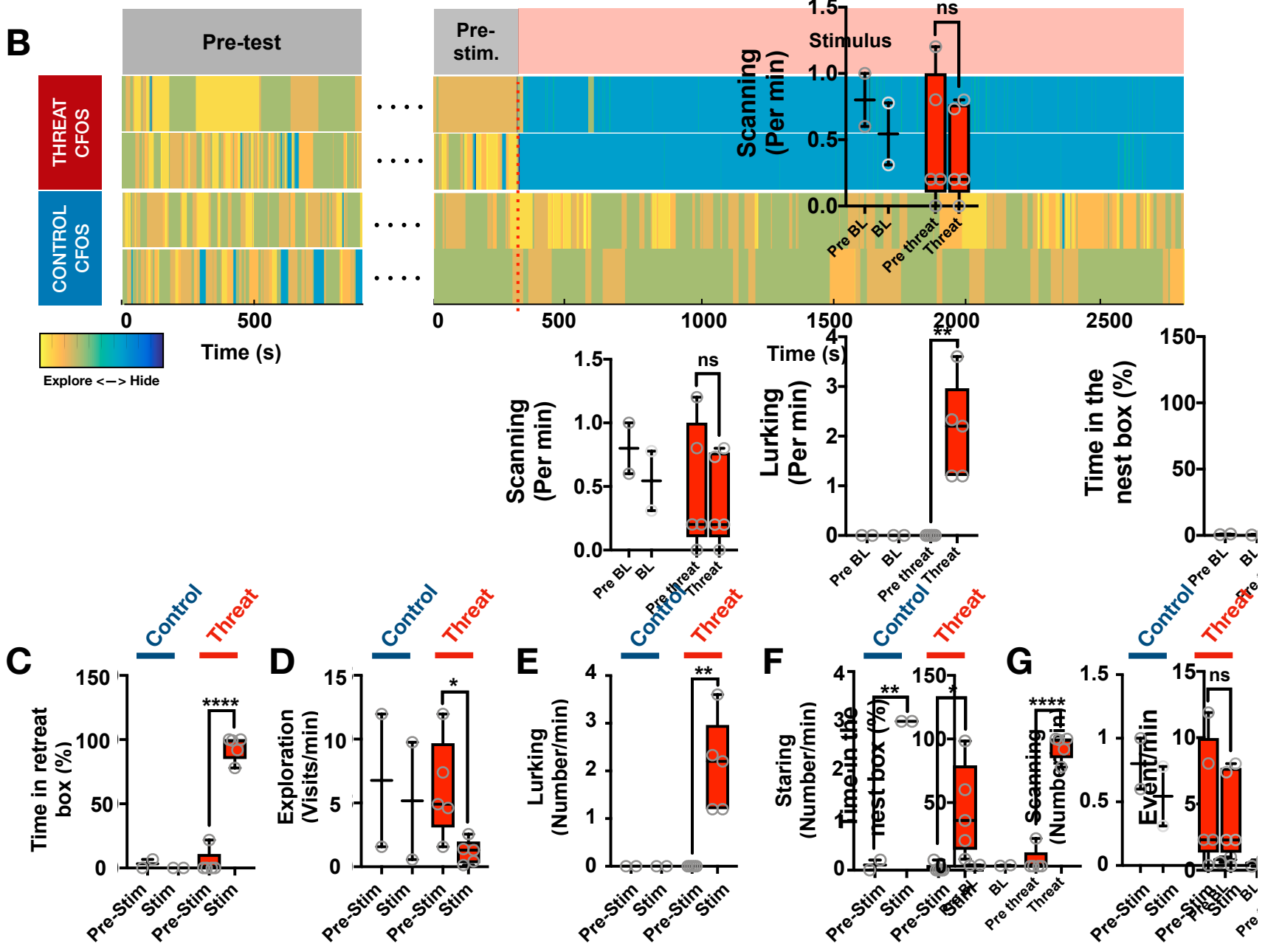


\section{Fig. S2}

A

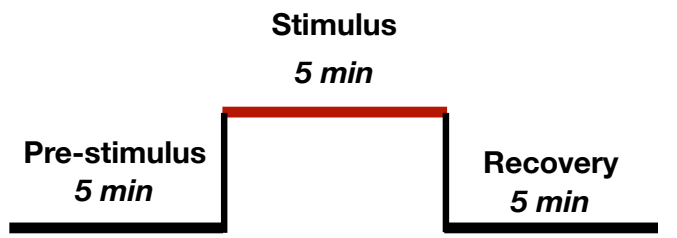

B

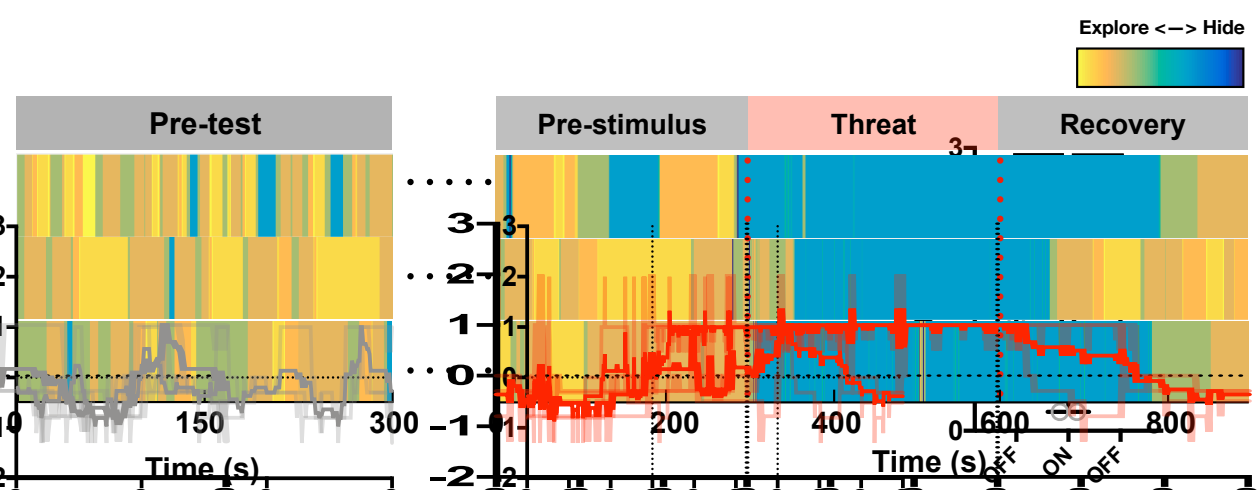

C
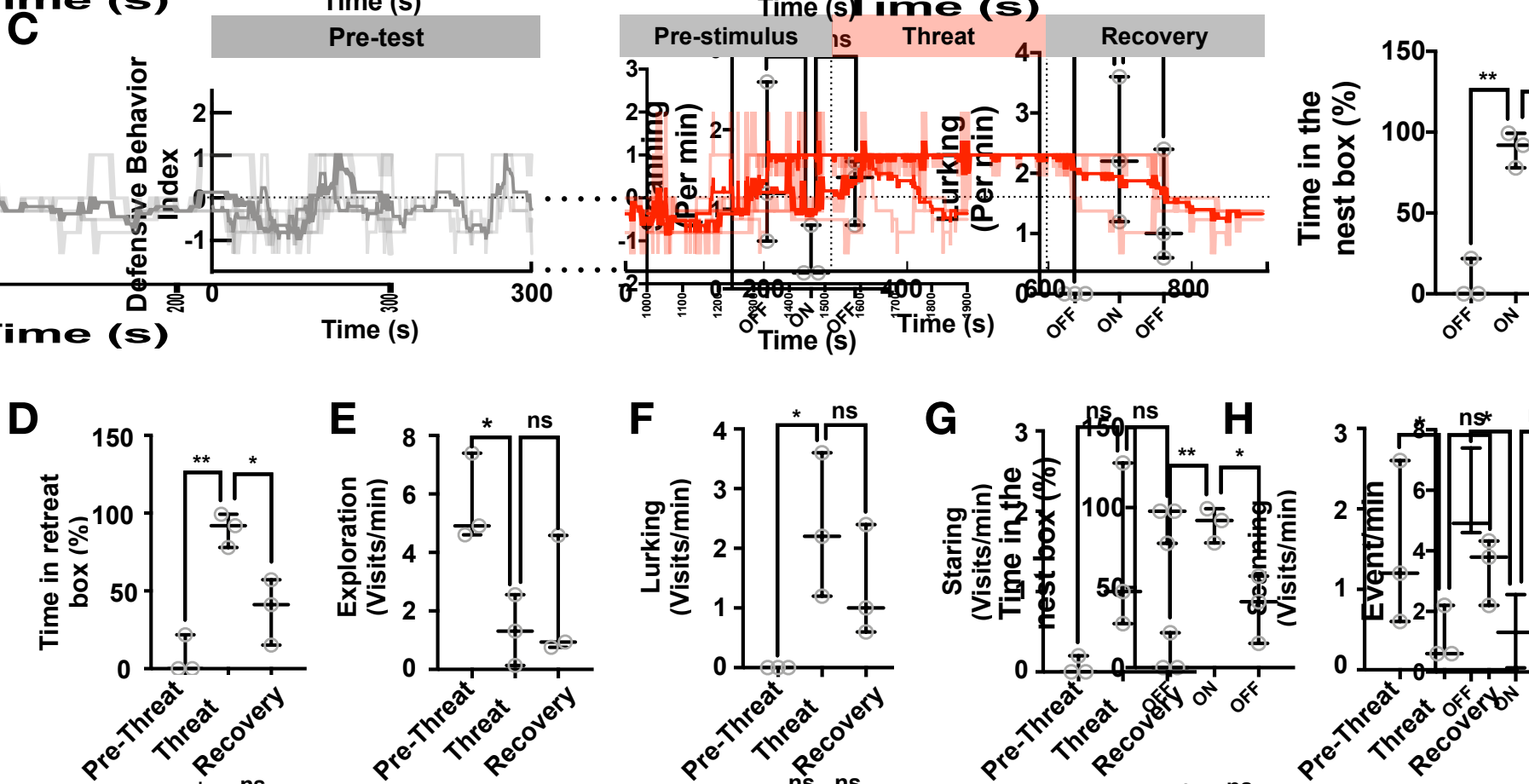


\section{Fig. S3}

A

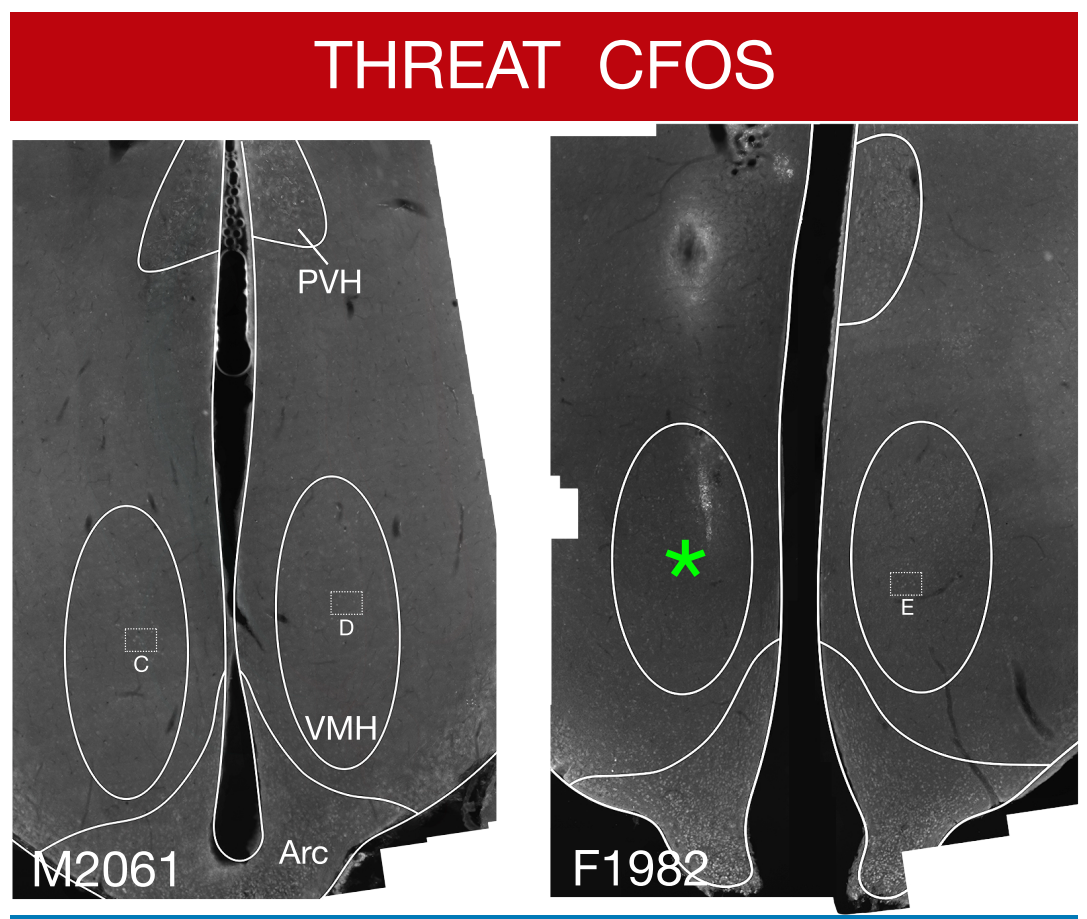

B

\section{CONTROL CFOS}
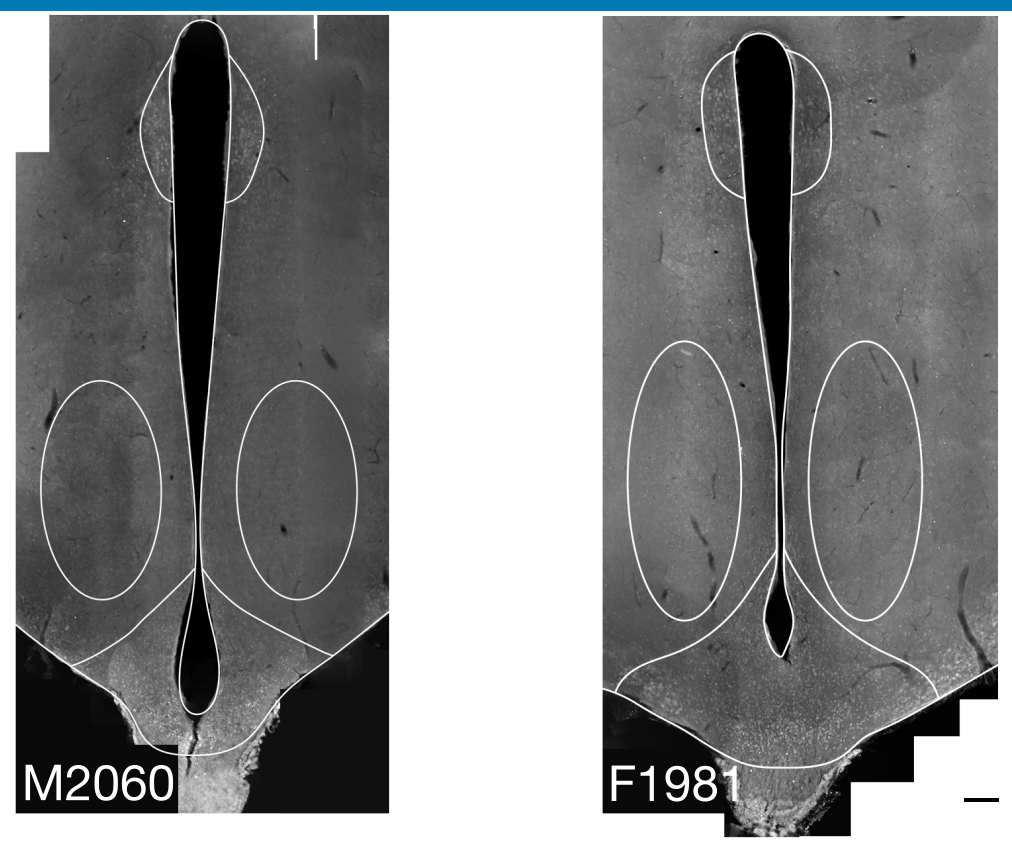

\section{C}
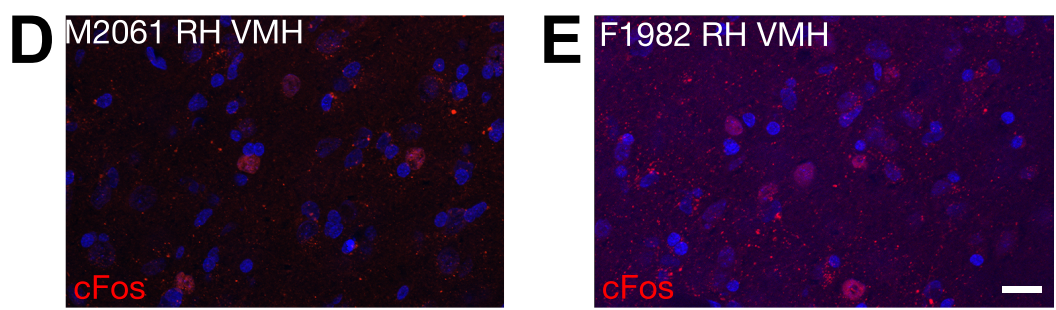


\section{Fig. S4}

\section{A}

\section{THREAT CFOS}

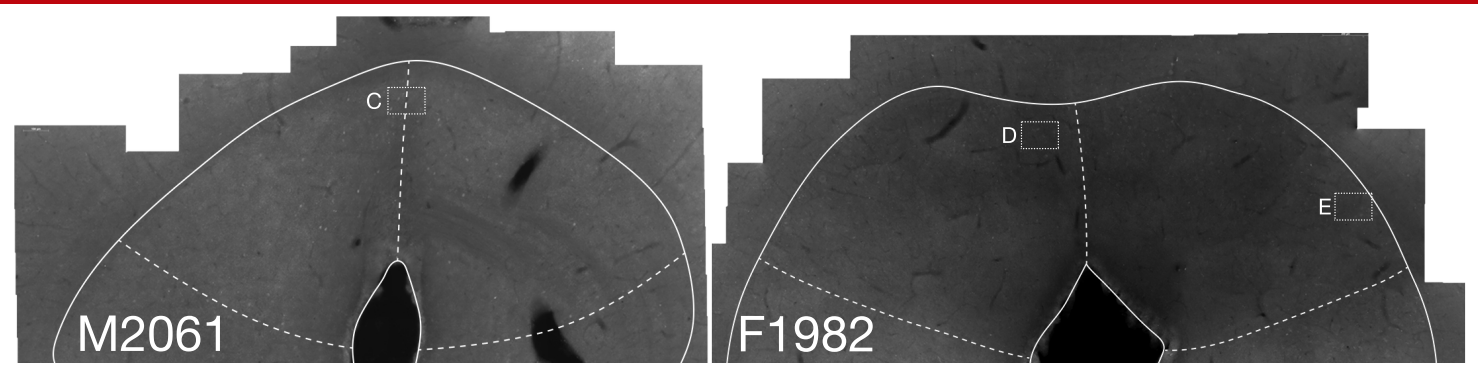

B

\section{CONTROL CFOS}
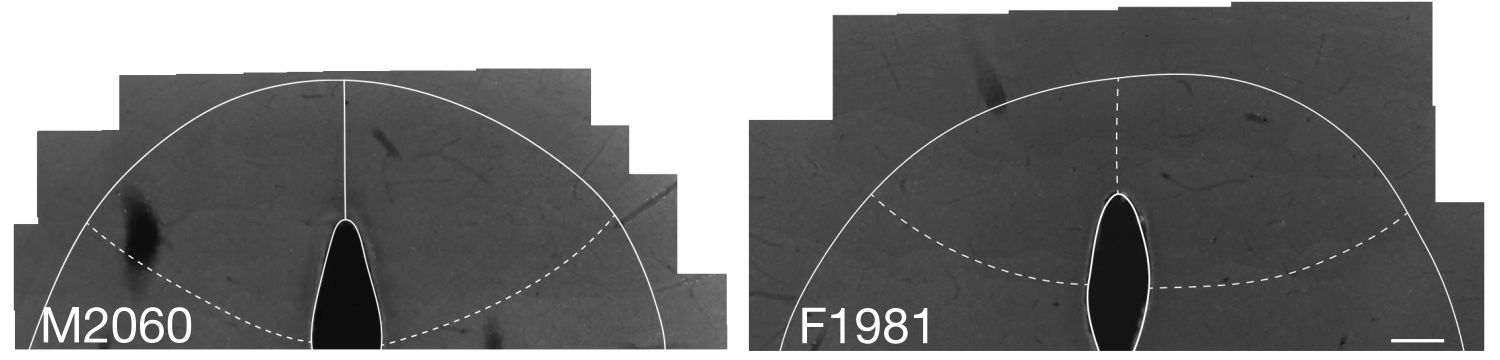

\section{C}

\section{M2061 dPAG}
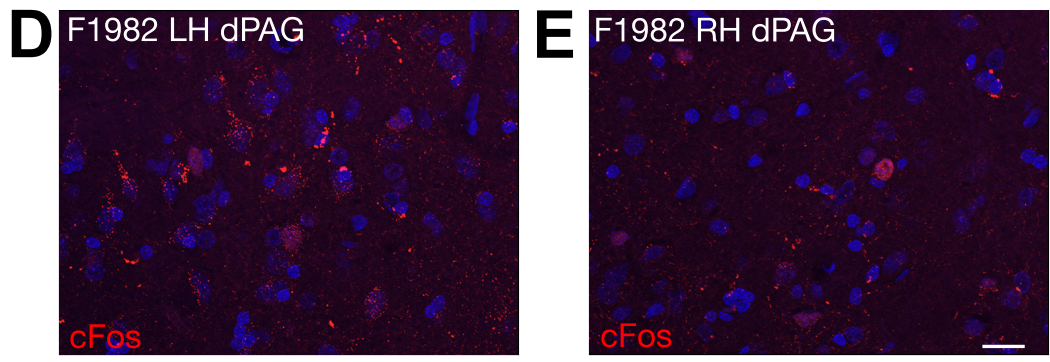
bioRxiv preprint doi: https://doi.org/10.1101/2020.04.24.057869; this version posted April 25, 2020. The copyright holder for this preprint (which was not certified by peer review) is the author/funder. All rights reserved. No reuse allowed without permission.

\section{Fig. S5}
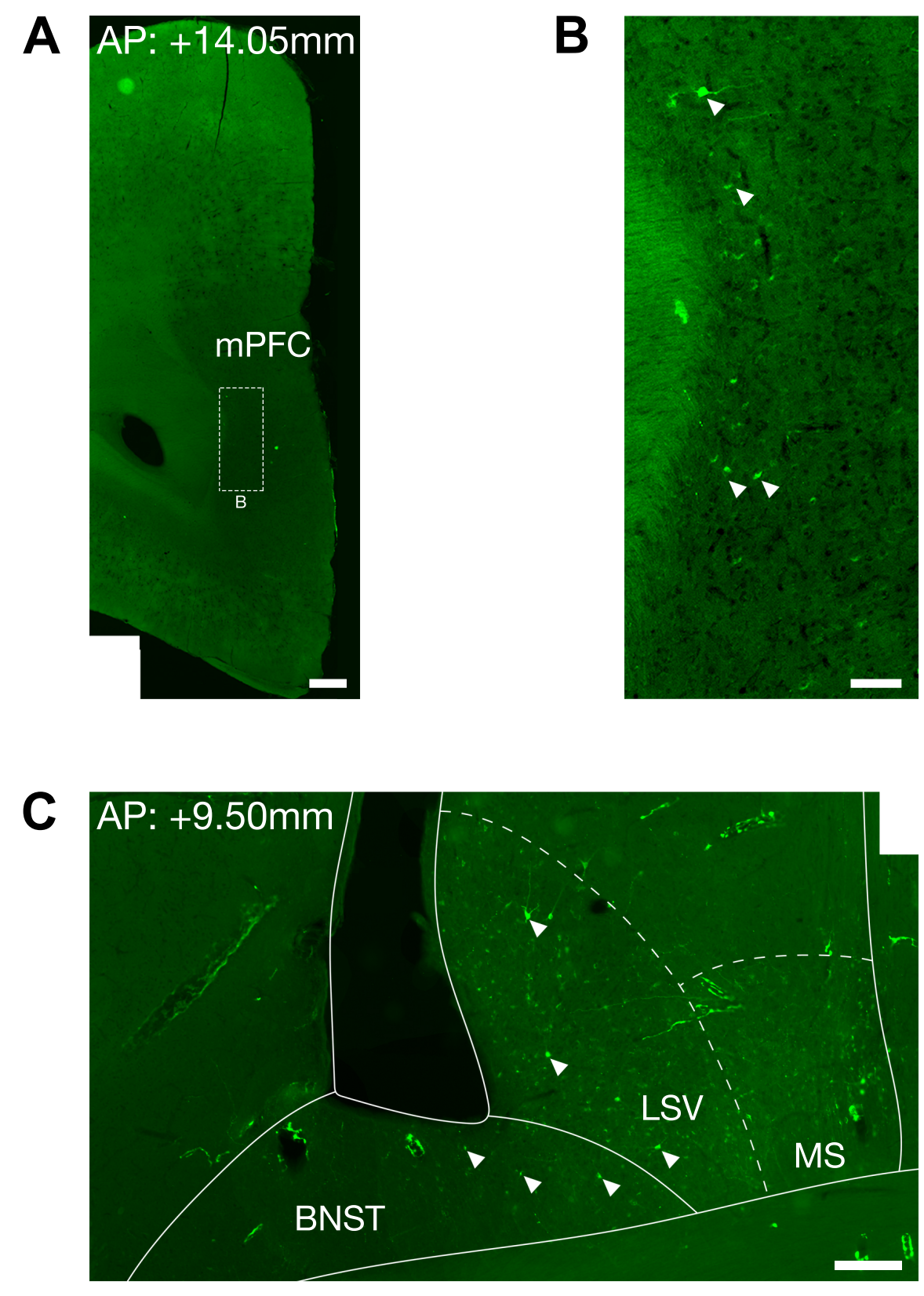
bioRxiv preprint doi: https://doi.org/10.1101/2020.04.24.057869; this version posted April 25, 2020. The copyright holder for this preprint (which was not certified by peer review) is the author/funder. All rights reserved. No reuse allowed without permission.

\section{Fig. S6}

A
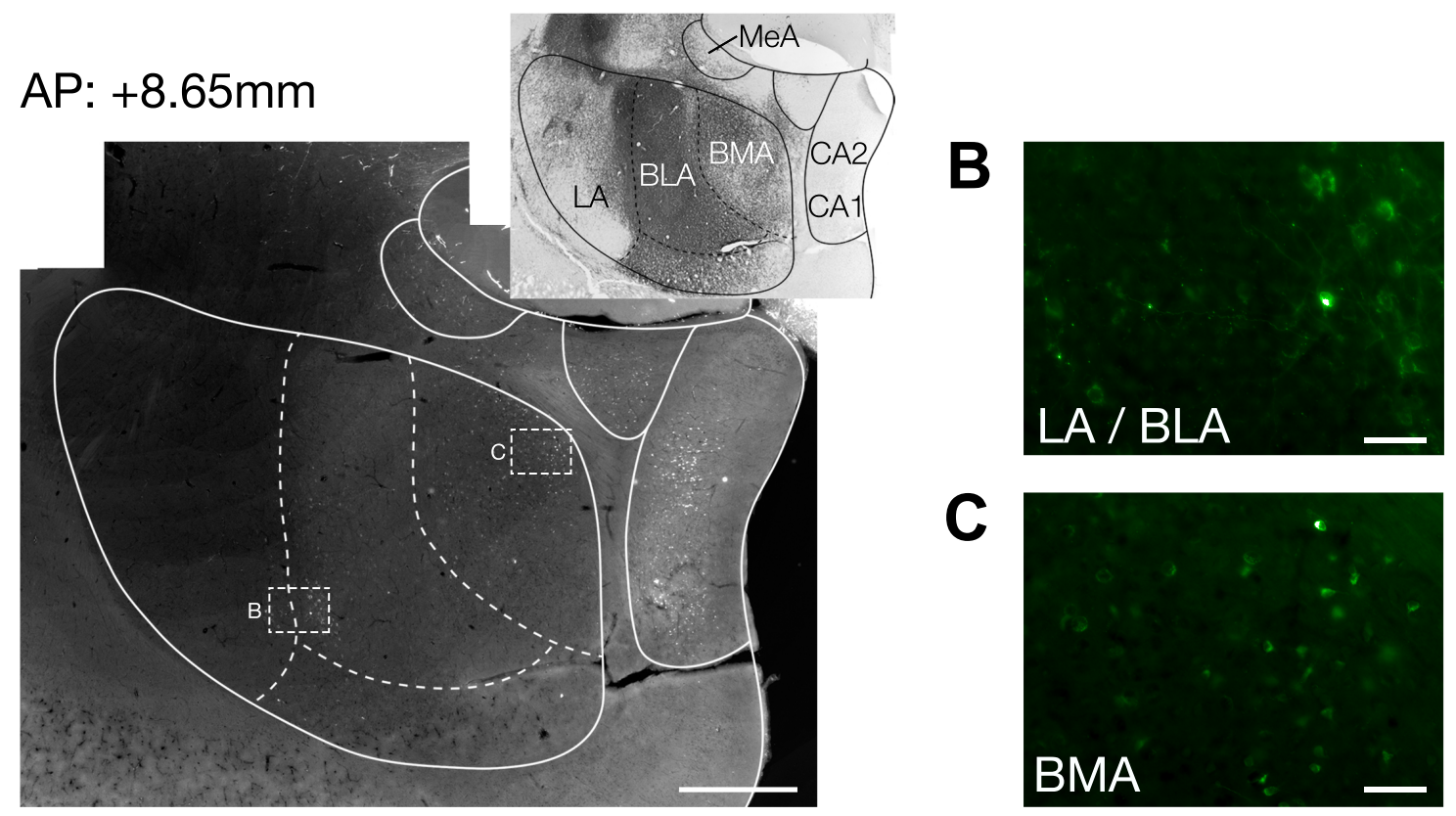

D

\section{$\mathrm{AP}:+9.15 \mathrm{~mm}$}
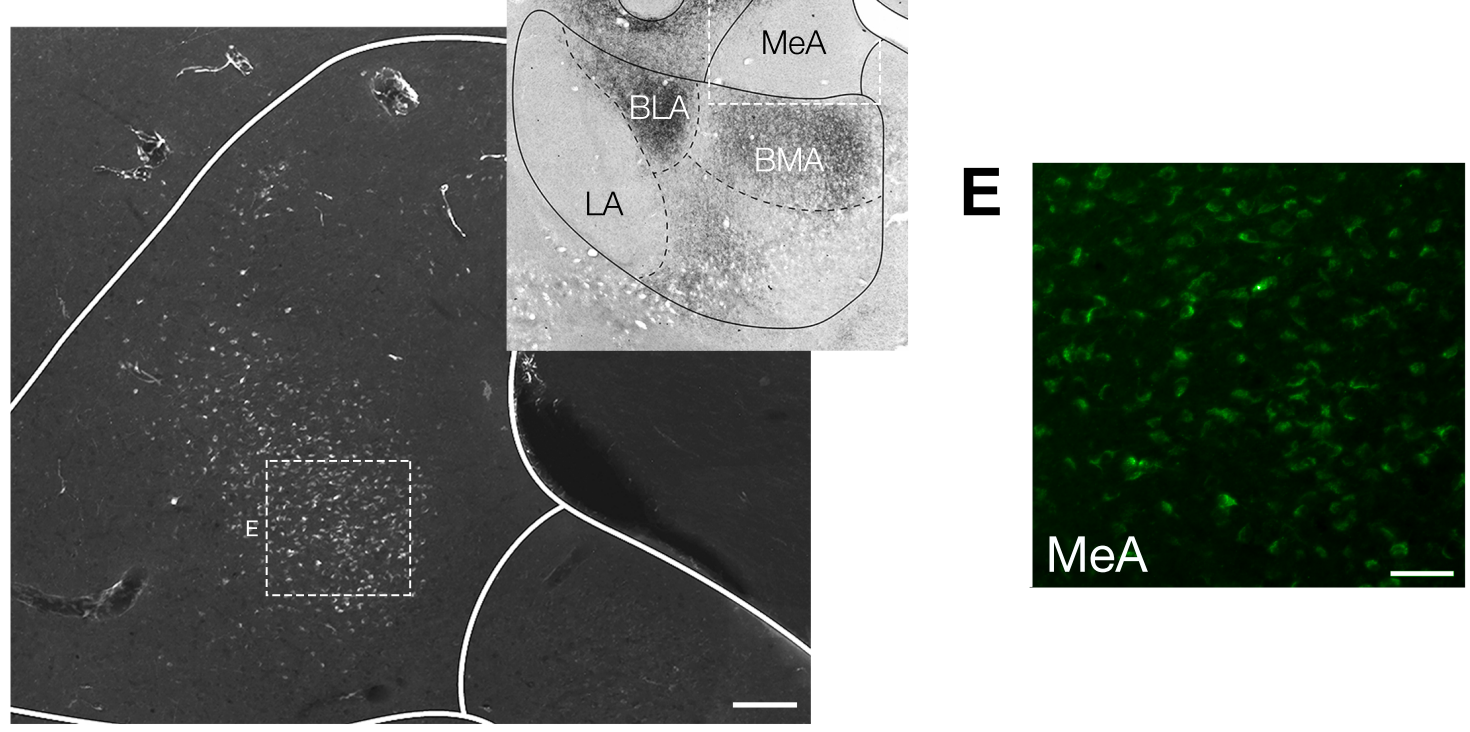
bioRxiv preprint doi: https://doi.org/10.1101/2020.04.24.057869; this version posted April 25, 2020. The copyright holder for this preprint (which was not certified by peer review) is the author/funder. All rights reserved. No reuse allowed without permission.

\section{Fig. S7}
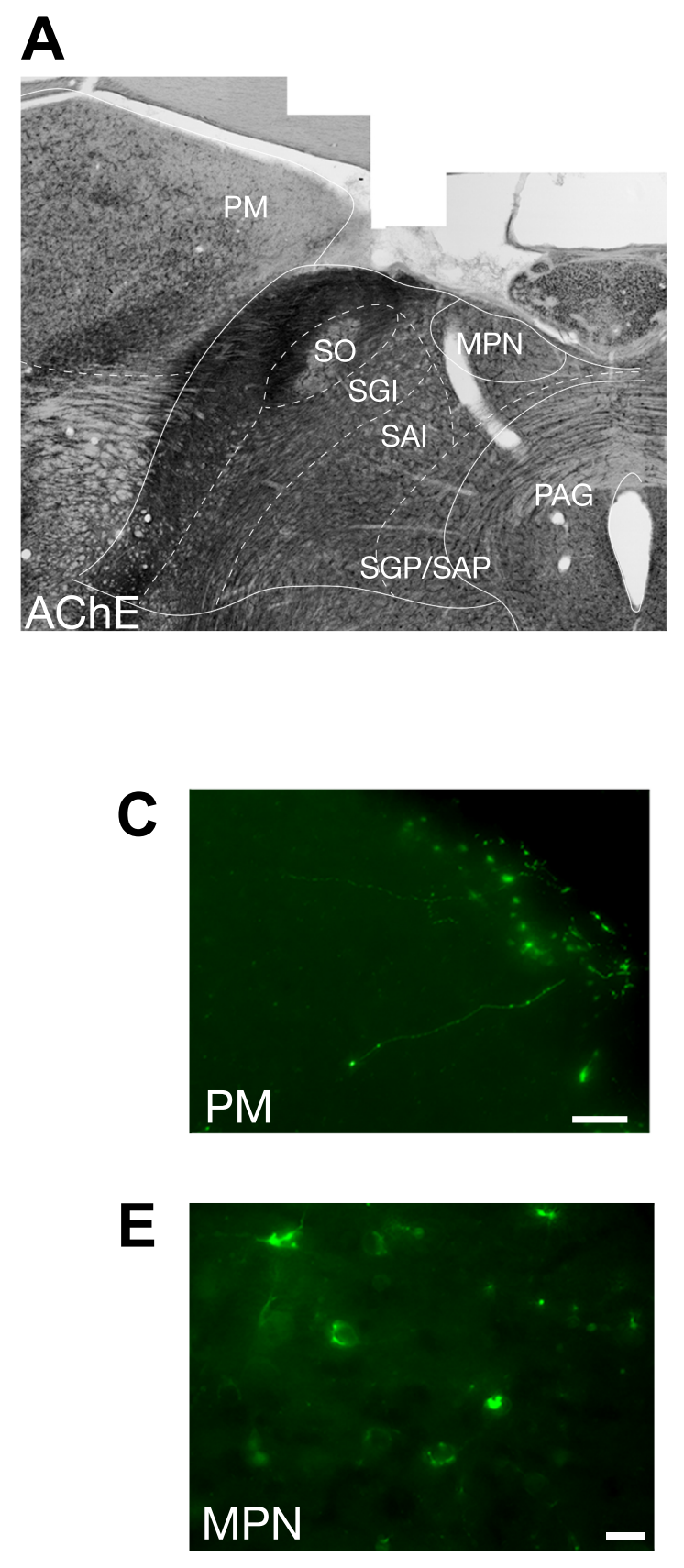

\section{B}
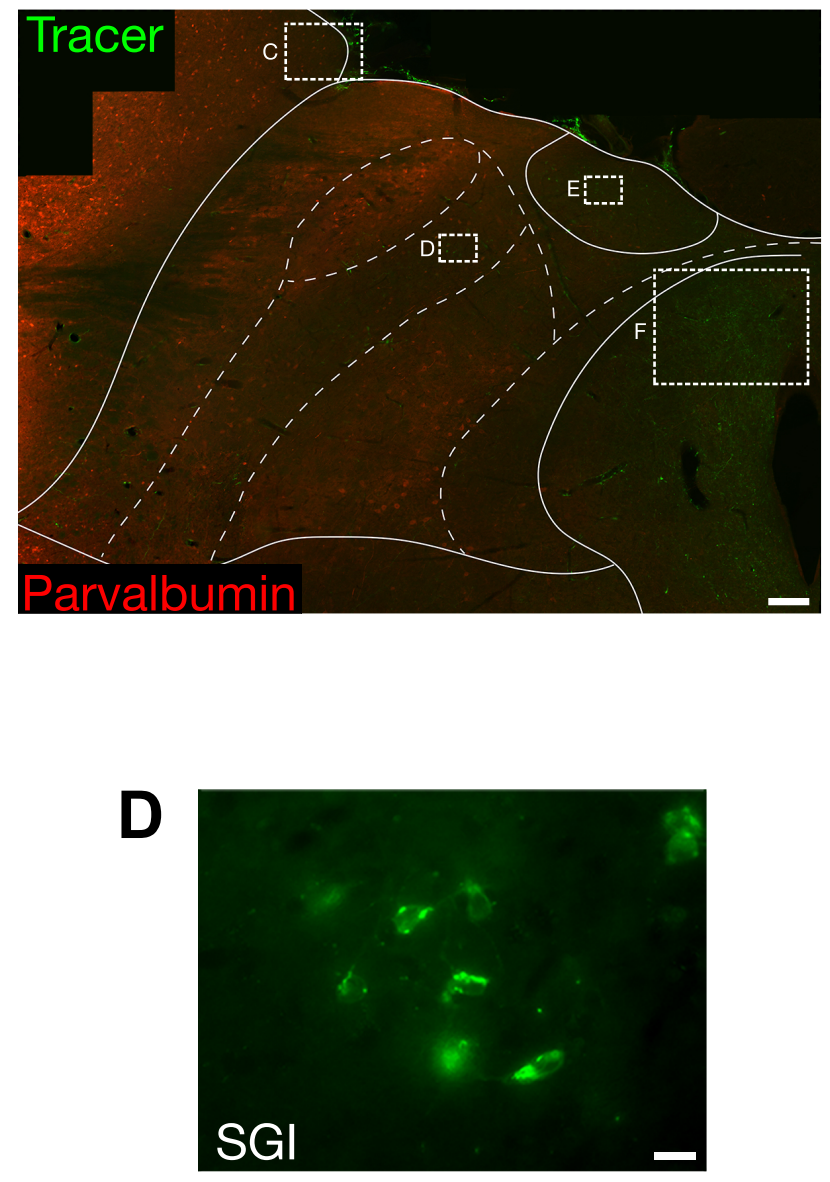

$\mathbf{F}$

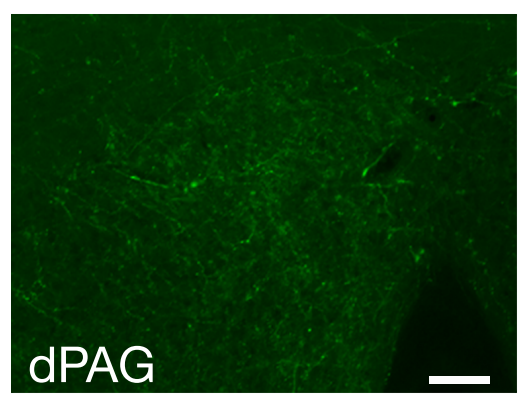

